\title{
Pneumoconiose em trabalhadores de carvoaria: um relato de caso
}

\author{
Pneumoconiosis in charcoal workers: a case report \\ Pneumoconiosis en trabajadores de carbón: un relato de caso
}

Bruno Peres Vilasboas Alves ${ }^{1}$,Mariana Teixeira Silveira Mendes Vilasboas Alves ${ }^{1}$, Emerson Willian Santos de Almeida ${ }^{2 \star}$, Frederico Marques Andrade ${ }^{2}$, João Marcus Oliveira Andrade ${ }^{2}$, Viviane Carrasco $^{2}$, Viviane Maia Santos ${ }^{1}$, Debora Ribeiro Vieira1, Daniel Oliva Brito ${ }^{1}$, Pedro Henrique Dias Cabral $^{2}$, Fernanda Amaral Soares Bicalho', Lanuza Borges Oliveira ${ }^{1,2}$.

\section{RESUMO}

Objetivo: Descrever a ocorrência de caso da pneumoconiose em trabalhador de uma carvoaria e os prejuízos a sua saúde e qualidade de vida. Método: Trata-se de um estudo tipo relato de caso durante atividades práticas do Curso de Pós-Graduação em Engenharia e Segurança do Trabalho Faculdades Integradas Pitágoras de Montes Claros no ano de 2015. Foi realizada entrevista com o trabalhador de uma carvoaria do interior do estado de Minas Gerais. Experiência: $O$ trabalhador tinha idade 72 anos, aposentado, ex tabagista, trabalhou por 7 anos em carvoarias do norte de Minas Gerais, na década de 90 cortando madeira, transportando a madeira até os fornos, abastecendo os fornos, acendendo e "apagando" os fornos e retirando o carvão em condições precárias segundo informações do paciente. Conclusão: Conclui-se que por meio do controle de monitoramento e prevenção haja a eliminação de riscos por meio de estratégias de treinamento e conscientização para o uso garantindo a qualidade de vida dos trabalhadores e prevenindo assim doenças como a PTC, pois a melhor medida para o controle é a prevenção a exposição.

Descritores: Carvão Vegetal, Pneumoconiose, Saúde do Trabalhador.

\begin{abstract}
Objective: To describe the occurrence of pneumoconiosis in a charcoal worker and the damages to his health and quality of life. Method: It is a study type a case report during practical activities of the Postgraduate Course in Engineering and Work Safety Faculdades Integradas Pitágoras de Montes Claros in the year 2015. An interview was made with the worker of a charcoal from the interior of the Minas Gerais state. Experience: The worker was 72 years old, retired, a former smoker, worked for 7 years in coal mines in the north of Minas Gerais, in the 1990s cutting wood, transporting the wood to the kilns, furnishing the kilns, lighting and "erasing" the furnaces and removing coal in poor conditions according to patient information. Conclusion: It is concluded that, through monitoring and prevention control, risk elimination can be achieved through training and awareness-raising strategies to ensure the quality of life of workers and thus prevent diseases such as PTC, since the best measure for the control is the prevention of exposure.
\end{abstract}

Keywords: Charcoal, Pneumoconiosis, Worker's health.

\section{RESUMEN}

Objetivo: Describir la ocurrencia de caso de la neumoconiosis en trabajador de una carbón y los perjuicios a su salud y calidad de vida. Método: Se trata de un estudio tipo un relato de caso durante actividades prácticas del Curso de Postgrado en Ingeniería y Seguridad del Trabajo. Facultades Integradas Pitágoras de Montes Claros en el año 2015. Se realizó una entrevista con el trabajador de una carvaje del interior del país en el estado de Minas Gerais. Experiencia: El trabajador tenía edad de 72 años, jubilado, ex tabaquista, trabajó

${ }^{1}$ Faculdades Integrada Pitágoras de Montes Claros (FIPMOC).

2 Universidade Estadual de Montes Claros (Unimontes). * E-mail: emerson93.ew@gmail.com

SUBMETIDO EM: $3 / 2018$

ACEITO EM: 4/2018

PUBLICADO EM: 1/2019 
por 7 años en carvoarias del norte de Minas Gerais, en la década de 90 cortando madera, transportando la madera hasta los hornos, abasteciendo los hornos, encendiendo y "apagando" hornos y retirando el carbón en condiciones precarias según la información del paciente. Conclusión: Se concluye que, a través del control de monitoreo y prevención, la eliminación de riesgos por medio de estrategias de entrenamiento y concientización para el uso garantizando la calidad de vida de los trabajadores y previniendo así enfermedades como la PTC, pues la mejor medida para el tratamiento el control es la prevención de la exposición.

Descriptores: Carbón Vegetal, Neumoconiosis, Salud del Trabajador.

\section{INTRODUÇÃO}

O contato do trabalhador com crosta terrestre ou poeiras de sílica implica diretamente ao quadro doença respiratória ocupacional com o maior índice de causa de invalidez, não possuindo tratamento especifico gerando complicações que poderão levar o paciente a morte. Portanto faz-se necessário que as empresas estabeleçam um ambiente de trabalho seguro com adequado monitoramento do ar e concentração de poeira (SANTOS et al, 2010).

As pneumoconioses não usuais, excetuando a silicose, a as bestose e a pneumoconiose do trabalhador do carvão se apresentam de maneira rara, entretanto, a dificuldade diagnostica demonstra a necessidade de anamnese ocupacional detalhada. É importante avaliação sistemática em relação as exposições ocupacionais, e em alguns casos, faz-se necessário o afastamento definitivo do trabalho (CAPITANI e ALGRANTI, 2006; SANTOS et al, 2010).

A pneumoconiose dos trabalhadores de carvão (PTC), se caracterizam por se tratar de uma doença pulmonar parenquimatosa causada pela inalação de fumaça na produção de carvão, atingindo as zonas distais do sistema respiratório e provocando reação tecidual do parênquima pulmonar. Os trabalhadores expostos podem apresentar tosse e produção de escarro, além da dispnéia associada a fibrose maciça progressiva que envolve o parênquima (TARANTINO, 2008)

Nas regiões onde se desenvolvem atividades por meio do corte, da carbonização e liberação de fumaça no ar que dependem da mão-de-obra humana, expõe os trabalhadores ao contato permanente com a fumaça oriunda dos fornos. Tal atividade expõe o trabalhador ao desenvolvimento da pneumoconiose podendo levar ao óbito (DIAS et al., 2002; LOPES, 2009).

A crescente ocorrência de internações hospitalares decorrentes da pneumoconioses tornou-se um problema de saúde pública, sendo necessário conhecer a sua realidade epidemiológica e realizar ações de controle, vigilância ambiental e prevenção dessas doenças no ambiente de trabalho (CASTRO et al., 2005).

Devido à ocorrência de casos da pneumoconiose entre trabalhadores de carvão e os prejuízos a saúde e qualidade de vida do trabalhador, este estudo objetivou descrever o relato de caso de um trabalhador com diagnóstico de pneumoconiose e os prejuízos a sua saúde e qualidade de vida.

\section{DETALHAMENTO DO CASO}

Trata-se de um estudo descritivo, tipo relato de caso a fim de descrever o caso de um trabalhador com diagnóstico de pneumoconiose e os prejuízos a sua saúde e qualidade de vida. Foi realizada entrevista com um Trabalhador de 72 anos, aposentado, ex tabagista, trabalhou por 7 anos em carvoarias do norte de Minas Gerais, na década de 90, em condições precárias segundo informações do paciente.

Seu trabalho era dividido entre cortar madeira, transportar a madeira até os fornos, abastecer os fornos, acender e "apagar" os fornos e retirar o carvão. O ciclo de um forno dura de seis a sete dias, como L.G.S descreveu. Inicialmente ocorria o acendimento do forno e o controle da entrada de ar, para que, pudesse ocorrer a carbonização da madeira. Após dois ou três dias, o forno era completamente vedado com argila e 
deixado em resfriamento até atingir temperaturas internas em torno de $40 \circ \mathrm{C}$ a $50 \circ \mathrm{C}$. O processo de acender e apagar os fornos levava em média 4 horas, e durante esse período L. G. S. ficava em contato direto com a grande quantidade de fumaça quente que saia dos fornos. O paciente relatou ainda que, por várias vezes sentia tonturas quando inalava uma grande quantidade de fumaça e, por muitas vezes não utilizava Equipamento de Proteção Individual (EPI).

Ao analisar prontuário médico, foi descoberto que, desde 1998, o paciente iniciou um ciclo de sintomas significativos, tosse produtiva constante, fadiga, dispnéia e dores no corpo que melhoravam parcialmente com medicações sintomáticas, mas que logo retornavam com certa periodicidade, fato que tornou o quadro clínico crônico. Os sintomas perduraram por 16 anos sem que o diagnóstico correto da pneumoconiose desse trabalhador fosse concluído.

Em meados de 2014, foram coletadas amostras de escarro para pesquisa de BAAR, cujo resultado positivo. Foi iniciado o tratamento para tuberculose pulmonar por seis meses, repetido BAAR cujo resultado negativo. Ao exame radiológico do tórax, observam-se extensas opacidades disseminadas, bilaterais, em terço médio e bases, que contribuíram para o diagnóstico da patologia discutida. A tomografia computadorizada de tórax apresentou aspecto multicístico pulmonar bilateral (Honeycomblung), mais acentuado a direita, com faveolamento periférico entremeado a bolhas enfisematosas para-septais, centroacinares, espessamento de septos interlobulares, sendo que nos lobos superiores há opacidades inflamatórias. Atualmente, o paciente segue em uso de anti-inflamatórios, analgésicos e suplementos vitamínicos. Mantém o quadro de dispnéia aos mínimos esforços, tosse e continua em acompanhamento clínico ambulatorial.

\section{DISCUSSÃO}

As principais características da pneumoconiose em trabalhadores de carvoaria se distingue pela formação de máculas pigmentadas peribronquiolares e perivasculares, as quais desenvolvem armazéns de reticulina, muitas vezes associadas com reação colágena focal e associações sob à presença de corpos birrefringentes à luz polarizada. Por sua vez, percebe-se que em sua fase inicial a doença não costuma apresentar sintomas, entretanto, muitos dos trabalhadores apresentam fibrose maciça progressiva (BRASIL, 2006). No prontuário de L.G.S não foi encontrado exame médico admissional, e/ou exames médicos periódicos, com avaliação clínica e exames complementares de acordo com os riscos da função inalada pelo trabalhador.

As formas iniciais ou simples da PTC, em geral, não apresentam sintomatologia e o exame clínico sem alterações. A PTC pode estar associada à Doença Pulmonar Obstrutiva Crônica, ao hábito de fumar e também a asma ocupacional. Nas formas avançadas, o paciente pode apresentar dispnéia aos mínimos esforços, tosse com catarro, diminuição do murmúrio vesicular, sibilos, roncos e com grandes opacidades à radiografia de tórax (TARANTINO, 2008). Além da dificuldade em concluir o diagnóstico da pnuemoconiose, percebe-se nesse estudo que esse fato afeta diretamente na qualidade de vida do trabalhador. O paciente em estudo por 16 anos sem o diagnóstico correto da doença.

Estudo observacional de caráter transversal realizado por Borges et al (2016) avaliando a função pulmonar e sintomas respiratórios em trabalhadores da mineração de pirocloro identificaram que dos 147 trabalhadores, todos eram do sexo masculino, com idade média de 41,37 \pm 8,71 anos apresentando uma média de 12,26 \pm 7,09 anos expostos à poeira. Em relação a identificação de doenças respiratórias foi encontrado que $22,44 \%$ apresentaram sintomas respiratórios e alguns chegaram a apresentar a presença de mais de um sintoma. Entretanto foi observado que, na avaliação da função $82,31 \%$ dos trabalhadores apresentaram valores espirométricos normais, sendo que $22,8 \%$ que apresentarão alterações no exame possuíam história de tabagismo ou ex-tabagismo. O tabagismo consequentemente aumenta a chance do surgimento de complicações no aparelho respiratório e reduz a função pulmonar, em relação a obstrução nasal em carvoeiros (SOUZA et al., 2010). 
Estudo realizado por lido et al., (2008) sobre a Exposição ocupacional e ocorrência de pneumoconioses na região de Campinas (SP) Brasil, 1978-2003, encontraram 43 tipos de ocorrências de casos de pneumoconioses sendo frequente em estampadores, forneiros, esmaltadores, nos que trabalham com peças de cerâmicas em fornos de curagem, moleiros e em operadores de moinho. Souza et al., (2010) evidenciaram em estudo que aqueles trabalhadores que residem a uma distância menor que $200 \mathrm{~m}$ da carvoaria possuem maiores riscos comparados aos que moram a distância superior a $200 \mathrm{~m}$.

Em outro estudo sobre o perfil epidemiológico de um grupo de Mineiros, pode-se perceber em relação as funções desenvolvidas pelos trabalhadores nas minas foi investigando 142 trabalhadores, os quais, possuem uma média de exposição em de 17,71 anos, conseguiram evidenciar por meio de exames de imagem classificações radiográficas, sendo que 113 trabalhadores não apresentaram alterações, 17 apresentaram exame suspeito e 7 com diagnostico de pneumoconiose confirmado com o resultado do exame de imagem (SOUZA et al., 2013).

Lopes (2009) afirma que, o diagnóstico clínico baseado na história de exposição e anormalidades encontradas em radiologias, não existem tratamento efetivo, deverá ser fornecido os cuidados de suporte e reabilitação apropriados.

Segundo Tarantino (2008), o pulmão pneumoconiótico predispõe à ocorrência de tuberculose. O trabalhador do caso relatado apresentou tuberculose, e com isso, houve o agravamento da fibrose pulmonar sem resposta ao tratamento. A maioria dos trabalhadores de carvoarias que apresentam problemas de saúde relacionados a essa atividade, são tratados como portadores de uma pneumopatia, em que, o profissional que o acompanha considera apenas os sintomas e não se atenta para as causas dos mesmos, este fato faz com que hajam poucos casos relatados e consequentemente uma subnotificação e o desconhecimento da gravidade da doença.

Filho (2015) em seu estudo sobre Agravos respiratórios em trabalhadores da construção civil em mato grosso, ressalta que, é de fundamental importância que os serviços especializados em engenharia de segurança e em medicina do Trabalho (SESMT) juntamente com as comissões internas de prevenção dos acidentes (CIPA), os quais, são constituídas por trabalhadores da própria instituição, assumam o compromisso na realização continua de monitoramento e prevenção de agravos no ambiente de trabalho reduzindo o número de doenças respiratórias.

De acordo com a norma regulamentadora 07 (sete) sobre o Programa de Controle Médico de Saúde Ocupacional, é obrigatória a realização de exames clínicos admissionais, periódicos, retorno ao trabalho, mudança de função e demissional. É imprescindível o acompanhamento médico periódico, não só a para prevenção, mas também para o acompanhamento da pneumoconiose (BRASIL, 1978; BRASIL, 2015).

Souza et al., (2013) afirmam em estudo sobre Análise documental do perfil epidemiológico de um grupo de mineiros da região Carbonífera do Extremo Sul Catarinense no ano de 2006 que, a redução de casos de Pneumoconiose nos mineiros de carvão estão relacionadas as boas condições no ambiente de trabalho, assim como o uso de EPI's e redução da poeira.

Portanto, conclui-se que, por meio do controle de monitoramento e prevenção haja a diminuição de riscos por meio da substituição ou adequação dos processos produtivos adotando medidas de proteção em nível coletivo e pessoal. Por sua vez é importante que empregador assuma a responsabilidade na entrega de EPIs e realize treinamentos e conscientizações para o uso adequado desses e assim proporcione qualidade de vida dos trabalhadores e prevenção de doenças como a PTC, uma vez que, a melhor medida para o controle é a prevenção a exposição.

\section{REFERÊNCIAS}

1. BORGES RCCO, JÚNIOR JCB, OLIVEIRA FB et al. Avaliação da função pulmonar e sintomas respiratórios em trabalhadores da mineração de pirocloro. J. bras. pneumol. São Paulo, 2016; 42 (4). 
2. Brasil. Ministério da Saúde. Secretaria de Atenção à Saúde. Departamento de Ações Programáticas Estratégicas. Pneumoconioses / Ministério da Saúde, Secretaria de Atenção à Saúde, Departamento de Ações Programáticas Estratégicas. - Brasília : Editora do Ministério da Saúde, 2006. 76 p. : il.

3. BRASIL. NR 31 - SEGURANÇA E SAÚDE NO TRABALHO NA AGRICULTURA, PECUÁRIA SILVICULTURA, EXPLORAÇÃO FLORESTAL E AQUICULTURA. Portaria MTE n.․ 86, de 03 de março de 2005.

4. BRASIL. NR 7 - PROGRAMA DE CONTROLE MÉDICO DE SAÚDE OCUPACIONAL. Portaria GM n.․ 3.214, de 08 de junho de 1978.

5. CAPITANI EM, ALGRANTI E . Outras pneumoconioses. J. bras. pneumol. São Paulo, 2006; 32 (I.2).

6. CASTRO HA, SILVA CG, VICENTIN G et al. Estudo das internações hospitalares por pneumoconioses no Brasil, 1984-2003. Rev. bras. epidemiol. São Paulo, 2005; 8(2).

7. LIDO AV, KITAMURA S, OLIVEIRA JI et al. Exposição ocupacional e ocorrência de pneumoconioses na região de Campinas (SP) Brasil, 1978-2003. J Bras Pneumol. 2008;34(6):367-372.

8. LOPES AC (Org.). Tratado de clínica médica. 2.ed. São Paulo: Roca, 2009. v.1.

9. MENDES, R. (Org.). Patologia do Trabalho. 2.ed. São Paulo: Atheneu, 2005. v.2

10. SANTOS C, NORTE A, FRADINHO F et al. Silicose - Breve revisão e experiência de um serviço de pneumologia. Rev Port Pneumol, Lisboa, 2010; 16 (1).

11. SOUZA FJFB, GALLAS MG, FILHO AJS. Análise documental do perfil epidemiológico de um grupo de mineiros da região Carbonífera do Extremo Sul Catarinense no ano de 2006. Arq Catarin Med. 2013 jul-set; 42(4): 73-78

12. SOUZA RM, ANDRADE FM, MOURA ABD et al. Sintomas respiratórios em trabalhadores de carvoarias nos municípios de Lindolfo Collor, Ivoti e Presidente Lucena, RS. J Bras Pneumol. 2010;36(2):210-217.

13. TARANTINO, A.B. Doenças pulmonares. 6.ed. Rio de Janeiro: Guanabara Koogan,2008. 